acknowledged that government publicity, particularly for distribution abroad, is essential.

D. E. Woodham

\section{Plant Chromosomes}

Chromosomal Evolution in Higher Plants. By Ledyard G. Stebbins. Pp. viii+216. (Arnold: London, March 1971.) $£ 4$ boards; $£ 2$ paper.

For a generation now of biologists, or more precisely botanists, the meaning of cytology has narrowed almost exclusively to the description of the condensations and movements of chromosomes in cell division. We have for many years taken it for granted that neither the evolution nor the ecology nor the taxonomy of plants can properly be discussed without relating them to the karyotypes. Consequently, and quite rightly, many botanists regard the handling of chromosomes as part of their stock in trade and for many it is very nearly their only pursuit, the only path to an understanding of the biology of plant populations. We owe this attitude to a very few who through their writings have come to be the interpreters of chromosome shapes and numbers; who, starting from the simple notion of the chromosome theory of heredity, have established the principles by which the chromosomes determine, or at any rate mark, the pathways of ecological adaptation and evolution. Professor Stebbins is one of them, and any book by him carries the print of authority.

This book is one in the Contemporary Biology series edited by E. J. W. Barrington and A. J. Willis and it is a shortish textbook aimed, I guess, at the first or second year student, although it must also become a useful handbook for more advanced students. Its style is straightforward and its contents are simple and arranged in a logical progression of ideas, starting with the function and organization of chromosomes and progressing through descriptions of the origin of variation in chromosome shape, size and number to the ecological and geographical consequences of karyotype variation. The book is plentifully illustrated, mostly with well chosen and well designed diagrams or clear drawings of chromosomes and karyotypes which are very easy to understand, and with maps and one or two photographs. There are also several informative tables.

A few minor criticisms can be made. Occasionally a technical term slips in which is not explained in the text or mentioned in the index. The discussion of heterochromatin I found interesting, but over-simplified. It left me with the impression that it is a material the function and properties of which can be described in a particular way, whereas it is, in fact, a term which can have almost as many meanings as there are people who use it. The rather narrow emphasis leads to some confusion later, when heterochromatin is treated as a disposable component of chromosomes to account (in the accepted manner) for changes in basic number. Rather more serious is a degree of confusion which arises from the author's efforts to treat the evidence for the correlation of karyotype evolution (asymmetry, for example) with ecological adaptation in a wholly critical manner. Inevitably, one is never certain whether the author wishes one to believe that morphological and ecological specialization results in or from asymmetry: whether it is causal, consequential, conditional or coincidental.

With the chapters on polyploidy, however, the author does very well. The treatment is lucid and he sweeps away, thank goodness, much of the rigid and stereotyped thinking on the subject that has been almost axiomatic for many years. It is quite clear from this section what role polyploidy has played in the generation of variants and the conservation of variation, in the spread and adaptation of populations. One derives from this account a clear impression of how plants evolve, and an insight into the complexity of the organization of plant populations as adapting breeding groups. Although Professor Stebbins sticks meticulously to the taxonomists' nomenclature the conviction must steal over any reader that, for example, the arbitrary notion of the species or genus as a definable level of biological organization is misleading and irrelevant, and that the key to understanding plants is not in classifying and naming them but in analysing their breeding systems. I have read more inspiring books on chromosomes, and more comprehensible ones, but there are not many which could give the non-specialist and biology student a neater account of chromosome evolution.

B. S. Cox

\section{Full Colour Pathology}

A Colour Atlas of General Pathology. By G. Austin Gresham. (Wolfe Medical Atlases-2.) Pp. 365. (Wolfe Medical: London, May 1971.) £3.75.

WHETHER the student beginning to study general pathology should use an atlas, an illustrated textbook or both is disputable. Teachers tend to favour textbooks, while students often like atlases and seem to benefit from them if they are relevant, and the pictures show clearly what they are meant to show and are accompanied by captions or explanatory sections. A justification of the atlas as a teaching aid is that students, while understanding much of what they read, are apt to have difficulty in identifying and interpreting what they see under the microscope. The atlas produced by Dr Gresham is a laboratory manual designed to supplement rather than replace the textbook. There are 435 colour photographs (chiefly $8 \times 5 \mathrm{~cm}$ ) with short explanatory captions on the facing page, and each group of between two and twenty or more pictures is preceded by a few paragraphs outlining the subject and introducing the student to the principal concepts of histopathology. The first section of the atlas is devoted to normal cells and tissues and includes brief discussion of electron microscopy, histochemistry, fiuorescence microscopy and autoradiography. Then follow sections on variations in cell growth and differentiation, and on cell damage. The greater part of the book deals with responses to injury, including inflammation and repair, and with patterns of disease including infection, trauma, vascular disturbances, allergy, intoxications, nutritional disturbances and neoplasia. There is a short set of pictures illustrating artefacts and the atlas ends with a glossary, list of further reading and index. It thus covers the topics usually dealt with in a course of general pathology.

The success of a book of this kind obviously depends very largely on the quality of the pictures, and most of these are good. There are several lapses, however, such as tissues stained with haematoxylin and eosin appearing dark blue-green (Figs. 151 and 152) or bright yellow (Figs. 396 and 416), emphasizing the fact that reproduction of photomicrographs still presents considerable problems. Some of the details described in the captions are difficult to see and a few arrows would help. The mixing of macroscopic pictures and photomicrographs adds interest to the illustrations.

The text is simple and direct, with a tendency to a vernacular style, and controversial statements are few. A future edition might benefit from an explanation of teratomas (illustrated but not discussed) and embryonal tumours, as well as a section on carcinogenesis illustrated with animal material. The number of printing errors is regrettably large. To give examples, the magnification of Fig. 237 is surely wrong, Figs. 388 and 389 are reversed, the heading to page 330 is truncated and the main index heading of Copper is omitted. There are irritating errors of punctuation on pages 247 and 281. "Lipoid" is used instead of the more usual "lipid" throughout.

I believe, however, that many students will like this book and benefit from it. The price is reasonable, especially taking the large number of colour pictures into account. The second of a series of atlases, it is to be followed, according to 